\title{
Vasospasm Versus Delayed Cerebral Ischemia as an Outcome Event in Clinical Trials and Observational Studies
}

\author{
Mervyn D. I. Vergouwen - The Participants in the International Multi-disciplinary \\ Consensus Conference on the Critical Care Management of Subarachnoid Hemorrhage
}

Published online: 12 July 2011

(C) Springer Science+Business Media, LLC 2011

\begin{abstract}
Delayed cerebral ischemia occurs in about 30\% of patients during the first 2 weeks after subarachnoid hemorrhage, and can result in substantial disability and death. Research studies investigating the incidence of delayed cerebral ischemia and strategies for prevention and treatment are hampered by inconsistent use of terminology and definitions for this complication, and by reliance on indirect surrogate markers of ischemia. A literature review was conducted to search for studies that addressed the issue of inconsistent definitions of delayed cerebral ischemia through December 2010. A total of four studies were identified. Original research studies and consensus panel recommendations for definitions support limiting the use of combined measures that include both clinical and radiographic assessments, as well as indirect measures, and the current usage of the term vasospasm. Cerebral infarction was supported as the most appropriate definition for delayed cerebral ischemia in the context of clinical trials.
\end{abstract}

The Participants in the International Multi-disciplinary Consensus Conference: Michael N. Diringer, Thomas P. Bleck, Nicolas Bruder, E. Sander Connolly, Jr., Giuseppe Citerio, Daryl Gress, Daniel Hanggi, J. Claude Hemphill, III, MAS, Brian Hoh, Giuseppe Lanzino, Peter Le Roux, David Menon, Alejandro Rabinstein, Erich Schmutzhard, Lori Shutter, Nino Stocchetti, Jose Suarez, Miriam Treggiari, MY Tseng, Mervyn D. I. Vergouwen, Paul Vespa, Stephan Wolf, Gregory J. Zipfel.

M. D. I. Vergouwen

Utrecht Stroke Center, Department of Neurology, University

Medical Center Utrecht, Heidelberglaan 100,

3584 CX Utrecht, The Netherlands

M. D. I. Vergouwen ( $\square)$

Department of Experimental Vascular Medicine, Academic

Medical Center, University of Amsterdam, Meibergdreef 9,

1105 AZ Amsterdam, The Netherlands

e-mail: M.D.I.Vergouwen@umcutrecht.nl
Keywords Angiography - Cerebral infarction · Clinical deterioration · Transcranial Doppler . Vasospasm

\section{Introduction}

Cerebral ischemia that occurs after subarachnoid hemorrhage (SAH) is called delayed cerebral ischemia (DCI). About $30 \%$ of in-hospital patients with aneurysmal SAH develop DCI as a complication within the first 2 weeks after the hemorrhage [1,2]. Clinical features of DCI can be reversible or progressing to cerebral infarction, which can result in long-lasting, severe disability or death.

Understanding the role of DCI after SAH is complicated by inconsistencies in definitions of DCI in the literature. In addition, a variety of terms have been used to reflect DCI (Box 1). In clinical trials and observational studies that have used DCI as an outcome event, terms and definitions to describe the phenomenon of cerebral ischemia have been inconsistently used [3, 4]. A major cause for this inconsistency is the utilization of definitions that combine radiographic evidence of vasospasm with clinical features of cerebral ischemia, considering that multiple factors may contribute to DCI [5-8]. A second cause of confusion is the variability and overlap of terms and definitions used to describe each phenomenon. In some cases, definitions are incomplete or absent. Inconsistencies in using terms and clarifying definitions make it almost impossible to compare the results between studies, understand the actual impact of treatment interventions, aggregate results into meta-analyses [9, 10], or construct guidelines with a high level of evidence $[3,11]$.

In order to appreciate the role of DCI in SAH, it is important to understand why the terms vasospasm and DCI are often used interchangeably and how they should be best 
Box 1 Terms used to describe ischemia occurred after SAH

- Delayed cerebral ischemia

- Delayed ischemic (neurological) deficit

- Secondary cerebral ischemia

- Symptomatic ischemia

- Vasospasm

- Clinical vasospasm

- Symptomatic vasospasm

- Cerebral infarction

defined: This review offers recommendations for definitions to most accurately express clinically important DCI as an outcome event in SAH research studies.

\section{Methods}

An electronic literature search was performed across the Pubmed database to look for the studies that addressed the issue of inconsistent definitions of delayed cerebral ischemia in human patients with SAH. Articles were identified using the combination of keywords "subarachnoid hemorrhage," "outcome measure," and "definition." Articles were included if they were published in English through December 2010.

\section{Summary of the Literature}

A total of 11 studies were retrieved, of which four were useful for the present review [3, 4, 12, 13]. These included one observational study, two review articles, and one consensus document.

\section{Defining Vasospasm}

Vasospasm is the observation of narrowing in intracranial arteries, resulting from vasoconstriction, swelling of the vascular endothelium, remodeling of the media and/or subendothelial fibrosis [14]. The Hagen-Poiseuille equation which shows that the total volume flowing through a tube varies according to the fourth power of a vessel's radius, supports the hypothesis that arterial narrowing results in reduced cerebral blood flow and a fall in oxygen delivery, eventually resulting in cerebral ischemia and infarction. Vasospasm can be visualized directly with angiography or indirectly by the observation of increased blood flow velocities on transcranial Doppler (TCD) examination. Vasospasm is sometimes already seen in SAH during the acute phase, but most commonly develops between days 4 and 14 after the hemorrhage, with a peak incidence at day 7.

Vasospasm Versus Delayed Cerebral Ischemia

Vasospasm is strongly associated with clinical deterioration due to DCI [15], cerebral infarction [16-18], poor outcome [18], and mortality [18] after SAH. Although clinical deterioration due to DCI is often seen in conjunction with angiographic evidence of vessel narrowing, each may occur independently of the other. Patients with SAH can also have clinical deterioration due to DCI in the absence of radiologically confirmed vasospasm [19, 20]. The reverse situation, where patients have angiographic vasospasm but no DCI, also occurs frequently [18, 19]. Arterial narrowing on angiography and increased blood flow velocities on TCD examination are often used as indirect, surrogate diagnostic markers for DCI for the following reasons: (1) clinical deterioration due to DCI is difficult to diagnose since it is a diagnosis per exclusionem (after exclusion of other causes, such as infection, hypotension, hyponatremia, etc.); (2) clinical deterioration due to DCI is difficult to diagnose in patients who are comatose or sedated; and (3) angiographic vasospasm is strongly associated with clinical deterioration due to DCI. This habit of relying on arterial narrowing has contributed to the interchangeable use of the terms DCI and angiographic vasospasm in many published studies.

\section{Outcome Measure for Trials}

Inconsistency in terms and definitions for vasospasm and DCI exist in the general, neurological, and neurosurgical literature [3, 4]. Two recent articles included in this review addressed the question of which definition of DCI is clinically the most useful $[12,13]$. In a large prospective cohort study including 580 patients from the Subarachnoid Hemorrhage Outcomes Project (SHOP) at Columbia University, DCI (defined as a new focal neurological deficit or decrease in level of consciousness, a new infarct revealed by followup imaging, or both) was independently associated with death or severe disability at 3 months only when computed tomography (CT) results were included in the definition of DCI [12]. Cerebral infarction alone had equally strong correlations with 3-month functional outcome (measured with either Glasgow Outcome Scale or modified Rankin Scale) as the combination of clinical deterioration due to DCI correlated with cerebral infarction on neuroimaging [21]. In the same cohort using a multivariate analysis, patients with asymptomatic infarction (mainly comatose patients) more often had a poor outcome (moderate-severe disability and death) compared with patients with clinical deterioration due to DCI [22]. It was found that neither the arterial narrowing 
observed during angiography nor the increased blood flow velocities measured with TCD were related to any aspect of clinical outcome.

Recently, a multidisciplinary research group proposed a uniform definition of DCI to be used as an outcome measure in clinical trials and observational studies [13]. The proposed definitions were partly based on the above described data from the SHOP registry $[12,21,22]$. In order to reduce inconsistencies in the use of definitions to describe DCI, it was suggested that DCI should not be defined by combining radiographic evidence of vasospasm with clinical features of cerebral ischemia. This consensus panel recommended using separately defined outcomes for angiographic vasospasm, clinical deterioration due to DCI, cerebral infarction, and functional outcome in clinical trials and observational studies. Cerebral infarction and functional outcome were recommended to be used as the two main outcome measures for studies aiming to reduce DCI rather than clinical deterioration due to DCI or angiographic vasospasm. Cerebral infarction on neuroimaging might be a better outcome measure than clinical deterioration due to DCI alone for several reasons. Cerebral infarction on CT or magnetic resonance (MR) imaging is (1) strongly correlated with functional outcome 3 months after SAH, (2) expected to have high interobserver agreement, (3) able to detect DCI in sedated and comatose patients, and (4) able to serve as a more objective quantification of the consequences of DCI. In addition, it has been shown that a lower incidence of cerebral infarction correlates with better functional outcomes after SAH [23]. Clinical deterioration due to DCI should be used as a secondary measure of outcome. Vasospasm on angiography can also be used as an outcome measure to investigate proof of concept, but should not be used as a surrogate outcome measure. Recently, in a meta-analysis of large multicenter trials, it was shown that a decreased incidence of vasospasm does not correlate with lower rates of poor functional outcome [24]. TCD examination has lower sensitivity and specificity to diagnose angiographic arterial narrowing [25], and therefore, it is not adequate to investigate proof of concept. It was strongly recommended to restrict the use of the words "vasospasm" and "arterial narrowing" to descriptions of radiological test results (e.g., CT or MR angiography, or digital subtraction angiography), and not to apply these terms to clinical manifestations of DCI. Recommended definitions for clinical deterioration and cerebral infarction due to DCI are provided in Boxes 2 and 3 .

\section{Conclusion}

Consistent use of valid terminology is essential for developing useful data about DCI after SAH. Cerebral infarction
Box 2 Proposed definition for clinical deterioration due to DCI [13]

- Focal neurological impairment (e.g., hemiparesis, aphasia, apraxia, hemianopia, or neglect)

- OR Glasgow Coma Scale reduction $\geq 2$ points with reductions occurring in the total score or on individual components (e.g., eye, motor on either side, or verbal)

- Deterioration should last $\geq 1 \mathrm{~h}$

- Abnormalities identified as deterioration should not have been apparent immediately after aneurysm occlusion and should not be attributable to other conditions

Box 3 Proposed definition of cerebral infarction from DCI [13]

- Cerebral infarction should be identified on CT or MR scan of the brain within 6 weeks after SAH, or on the latest CT or MR scan made before death within 6 weeks after SAH, or proven at autopsy

- Cerebral infarction should not have been present on CT or MR scan performed between 24 and $48 \mathrm{~h}$ after aneurysm occlusion

- Cerebral infarction should not be able to be attributed to other causes (e.g., surgical clipping or endovascular treatment)

- Hypodensities on CT imaging resulting from ventricular catheter or intraparenchymal hematoma should not be regarded as cerebral infarctions from DCI

on imaging studies or autopsy is the most appropriate measure of DCI in clinical trials and observational studies. Clinical deterioration due to DCI should be described separately from the results of angiography. Furthermore, the term vasospasm should be reserved for angiographic arterial narrowing only.

\section{References}

1. Roos YB, de Haan RJ, Beenen LF, Groen RJ, Albrecht KW, Vermeulen M. Complications and outcome in patients with aneurysmal subarachnoid haemorrhage: a prospective hospital based cohort study in the Netherlands. J Neurol Neurosurg Psychiatry. 2000;68:337-41.

2. Hijdra A, Van Gijn J, Stefanko S, Van Dongen KJ, Vermeulen M, Van Crevel H. Delayed cerebral ischemia after aneurysmal subarachnoid hemorrhage: clinicoanatomic correlations. Neurology. 1986;36:329-33.

3. van der Schaaf IC, Ruigrok YM, Rinkel GJ, Algra A, van Gijn J. Study design and outcome measures in studies on aneurysmal subarachnoid hemorrhage. Stroke. 2002;33:2043-6.

4. van Gijn J, Bromberg JE, Lindsay KW, Hasan D, Vermeulen M. Definition of initial grading, specific events, and overall outcome in patients with aneurysmal subarachnoid hemorrhage. A survey. Stroke. 1994;25:1623-7.

5. Grubb RL Jr, Raichle ME, Eichling JO, Gado MH. Effects of subarachnoid hemorrhage on cerebral blood volume, blood flow, and oxygen utilization in humans. J Neurosurg. 1977;46:446-53.

6. Uhl E, Lehmberg J, Steiger HJ, Messmer K. Intraoperative detection of early microvasospasm in patients with subarachnoid hemorrhage by using orthogonal polarization spectral imaging. Neurosurgery. 2003;52:1307-15.

7. Vergouwen MD, Vermeulen M, Coert BA, Stroes ES, Roos YB. Microthrombosis after aneurysmal subarachnoid hemorrhage: an 
additional explanation for delayed cerebral ischemia. $\mathrm{J}$ Cereb Blood Flow Metab. 2008;28:1761-70.

8. Dreier JP, Major S, Manning A, et al. Cortical spreading ischaemia is a novel process involved in ischaemic damage in patients with aneurysmal subarachnoid haemorrhage. Brain. 2009;132:1866-81.

9. Sillberg VA, Wells GA, Perry JJ. Do statins improve outcomes and reduce the incidence of vasospasm after aneurysmal subarachnoid hemorrhage: a meta-analysis. Stroke. 2008;39:2622-6.

10. Kramer AH. Statins in the management of aneurysmal subarachnoid hemorrhage - not (yet) a standard of care. Stroke. 2009;40:e80-1.

11. Bederson JB, Connolly ES Jr, Batjer HH, American Heart Association, et al. Guidelines for the management of aneurysmal subarachnoid hemorrhage: a statement for healthcare professionals from a special writing group of the Stroke Council, American Heart Association. Stroke. 2009;40:994-1025.

12. Frontera JA, Fernandez A, Schmidt JM, et al. Defining vasospasm after subarachnoid hemorrhage: what is the most clinically relevant definition? Stroke. 2009;40:1963-8.

13. Vergouwen MD, Vermeulen M, van Gijn J, et al. Definition of delayed cerebral ischemia after aneurysmal subarachnoid hemorrhage as an outcome event in clinical trials and observational studies: proposal of a multidisciplinary research group. Stroke. 2010;41:2391-5.

14. Hughes JT, Schianchi PM. Cerebral artery spasm. A histological study at necropsy of the blood vessels in cases of subarachnoid hemorrhage. J Neurosurg. 1978;48:515-25.

15. Fisher CM, Roberson GH, Ojemann RG. Cerebral vasospasm with ruptured saccular aneurysm-the clinical manifestations. Neurosurgery. 1977;1:245-8.

16. Rabinstein AA, Friedman JA, Weigand SD, et al. Predictors of cerebral infarction in aneurysmal subarachnoid hemorrhage. Stroke. 2004;35:1862-6.

17. Fergusen S, Macdonald RL. Predictors of cerebral infarction in patients with aneurysmal subarachnoid hemorrhage. Neurosurgery. 2007;60:658-67.
18. Vergouwen MDI, Ilodigwe D, Macdonald RL. Cerebral infarction after subarachnoid hemorrhage contributes to poor outcome by vasospasm-dependent and -independent effects. Stroke. 2011; 42:924-9.

19. Dankbaar JW, Rijsdijk M, van der Schaaf IC, Velthuis BK, Wermer MJ, Rinkel GJ. Relationship between vasospasm, cerebral perfusion, and delayed cerebral ischemia after aneurysmal subarachnoid hemorrhage. Neuroradiology. 2009;51:813-9.

20. Dankbaar JW, de Rooij NK, Velthuis BK, Frijns CJ, Rinkel GJ, van der Schaaf IC. Diagnosing delayed cerebral ischemia with different CT modalities in patients with subarachnoid hemorrhage with clinical deterioration. Stroke. 2009;40:3493-8.

21. Kreiter KT, Mayer SA, Howard G, et al. Sample size estimates for clinical trials of vasospasm in subarachnoid hemorrhage. Stroke. 2009;40:2362-7.

22. Schmidt JM, Wartenberg KE, Fernandez A, et al. Frequency and clinical impact of asymptomatic cerebral infarction due to vasospasm after subarachnoid hemorrhage. J Neurosurg. 2008;109: $1052-9$.

23. Vergouwen MD, Etminan N, Ilodigwe D, Macdonald RL. Lower incidence of cerebral infarction correlates with improved functional outcome after aneurysmal subarachnoid hemorrhage. J Cereb Blood Flow Metab. 2011;31:1545-53.

24. Etminan N, Vergouwen MD, Ilodigwe D, Macdonald RL. Effect of pharmaceutical treatment on vasospasm, delayed cerebral ischemia, and clinical outcome in patients with aneurysmal subarachnoid hemorrhage: a systematic review and meta-analysis. J Cereb Blood Flow Metab. 2011;31:1443-51.

25. Lee JY, Lee MS, Whang K, Lee JM, Kim SH, Lee SS. Accuracy of transcranial Doppler sonography for predicting cerebral infarction in aneurysmal subarachnoid hemorrhage. J Clin Ultrasound. 2006;34:380-4. 